\title{
Mapeo sistemático de la literatura sobre la eficacia colectiva docente
}

\author{
Fátima SALAS-RODRÍGUEZ \\ Sonia LARA
}

Datos de contacto:

Fátima Salas-Rodríguez Departamento de Aprendizaje y Currículum. Facultad de Educación y Psicología. Universidad de Navarra (España)

fsalas@alumni.unav.es

Sonia Lara

Departamento de Aprendizaje y Currículum. Facultad de Educación y Psicología. Universidad de Navarra (España)

slara@unav.es 


\title{
Systematic mapping of literature on collective teacher efficacy
}

\begin{abstract}
In recent years, collective teacher efficacy has been presented as a promising factor in improving student performance (Donohoo, 2017; Eells, 2011; Hattie, 2016). However, unlike self-efficacy beliefs, collective teacher efficacy has been studied much less. Hence, this systematic literature mapping seeks to deepen the development and study of the collective efficacy beliefs of teachers in the academic field, with the objective of understanding and synthesizing the information of various investigations regarding the subject in question. Through the methodology of systematic mapping, a total of 60 potential studies published between 2000 and 2019 and indexed in the Scopus and Web of Science databases were found. After the application of the inclusion and exclusion criteria, 35 studies were included in the present investigation. The results show the geographical distribution of the studies, the most frequently cited articles, the variables related to collective teacher efficacy, the instruments used to evaluate these beliefs, and the educational levels in which they have been studied. There is a clear interest in the study regarding the impact of collective teacher efficacy on student learning and performance. However, there is a lack of research on this subject in Spanish-speaking countries, as well as research focused on the promotion and development of collective teacher efficacy. This study concludes with a proposal for future lines of research.
\end{abstract}

KEYWORDS: Collective teacher efficacy; Teacher self-efficacy; Systematic mapping; Teachers; Educational improvement

\section{Introducción}

¿Cuál es la meta en educación? Ante esta pregunta surgen diversas respuestas: desarrollar las habilidades y destrezas del alumno; lograr que el discente aprenda una serie de contenidos y competencias; ayudar a que el educando se desarrolle y alcance la mejor versión de sí mismo; enseñar al alumno a vivir en sociedad; etc. Las metas y los objetivos en el ámbito de la educación formal son muchos y muy diversos, aunque no cabe duda de que el énfasis se ha puesto en el campo del aprendizaje y la adquisición del conocimiento. En todo caso, los diversos objetivos coinciden en que el receptor del proceso educativo es el alumno.

A pesar de tener esto claro, los medios y los procesos para alcanzar el desarrollo y aprendizaje del discente son muchos y muy variados. Por este motivo, a lo largo de los años, se han estudiado los diversos factores presentes en el proceso de enseñanza-aprendizaje, así como los diferentes sujetos en él implicados: alumnos, profesores, padres de familia y 
directores. En el 2007 la consultora McKinsey publicó el famoso informe sobre "Cómo hicieron los sistemas educativos con mejor desempeño del mundo para alcanzar sus objetivos" (Barber \& Mourshed, 2007). Una de las principales ideas que ahí se defienden es que la calidad de un sistema educativo tiene como techo la calidad de sus docentes; argumentando que los sistemas educativos con mejor rendimiento se caracterizan por atraer y seleccionar a los mejores candidatos para la profesión docente. De esta manera, se pone especial hincapié en el profesorado y en su capacidad como individuos para alcanzar el éxito educativo.

En este sentido, las políticas educativas de los últimos años se han caracterizado por la búsqueda y promoción del desarrollo y perfeccionamiento de los profesores como agentes individuales. De ahí el interés creciente por temas como la formación efectiva del profesorado (Darling-Hammond, Hyler, \& Gardner, 2017), el aumento de exigencia en la selección de futuros docentes, el estudio de la calidad docente individual mediante modelos de valor añadido, entre otros. Sin embargo, a pesar de que "gran parte del debate sobre la mejora de la enseñanza se centra en maestros efectivos como individuos, [...] las escuelas exitosas aumentan el rendimiento a través del trabajo en equipo" (Stewart, 2018, pp. 33-34 [traducción propia]). A este respecto Hargreaves y Fullan (2014) afirman que

El maestro es la clave. Pero esto no significa que deberíamos centrarnos en conseguir recompensar mejor a los maestros individuales. Los sistemas de rendimiento más elevados del mundo tienen buenos profesores, pero los tienen en cantidad. [...] Es cosa de la escuela, del profesional y del sistema. (p. 45)

En esta línea, Martínez Orbegozo $(2019$, p. 4) asegura que "el efecto total de un centro es mayor que la suma de los efectos individuales de sus docentes". Por este motivo, la calidad de un sistema educativo no puede tener como techo la calidad de sus docentes, sino más bien la calidad de sus centros. Así pues, si se quiere conseguir un cambio real a nivel educativo, el interés por la calidad docente debe de ir acompañado del apoyo y la mejora a nivel organizacional.

Quizá nos pueda servir de ejemplo el estudio de la eficacia colectiva docente. Esta variable fue nombrada por John Hattie (2016) como el factor con mayor impacto en el rendimiento del alumno, obteniendo un tamaño del efecto de 1.57 y dejando por debajo a variables como las expectativas del alumno (1.33), las discusiones en clase (0.82), el feedback (0.70), el rendimiento anterior del discente $(0.55)$, los programas de formación profesional (0.37), entre otras. Lo que sorprende de este listado es que la variable con mayor impacto en el aprendizaje de los alumnos es una variable colectiva a nivel de centro.

Dichas creencias colectivas se fundamentan en las creencias de 
autoeficacia, es decir, en los juicios sobre la propia capacidad para organizar y realizar determinadas acciones (Bandura, 1986, 1997). Estas creencias guían la propia conducta al influir en las elecciones que se toman, y en el esfuerzo y persistencia con que se realizan las acciones (Bandura, 1997). A mayor autoeficacia percibida, mayores serán los riesgos a tomar, el esfuerzo invertido y la persistencia implicada. Es importante dejar claro que las creencias de autoeficacia no se refieren a las habilidades que uno posee, sino a los juicios que uno hace con respecto a lo que puede llegar a realizar con dichas habilidades. De esta manera, para alcanzar un rendimiento adecuado se necesita tanto de la capacidad para realizar dicha acción, como de la creencia de que se dispone de la eficacia suficiente para utilizarla (Bandura, 1986).

En el ámbito educativo, la autoeficacia docente se conoce como la creencia que el profesor guarda con respecto a su capacidad para producir un cambio e influir en el desempeño de sus alumnos (Donohoo, 2017). Según Gibson y Dembo (1984) las creencias que tienen los docentes sobre la propia capacidad para enseñar a los alumnos, producen diferencias entre ellos a la hora de ser más o menos efectivos. Bandura (1993) afirma que los profesores con creencias elevadas sobre su eficacia crean experiencias de aprendizaje más ricas y complejas para sus alumnos, fomentando a su vez la autoeficacia de estos últimos. Asimismo, los estudios TALIS (Teaching and Learning International Survey) -estudios internacionales promovidos por la OCDE con el objetivo de conocer los entornos de aprendizaje y enseñanza desde la perspectiva del profesoradohan encontrado que las creencias de autoeficacia de los profesores se relacionan con la satisfacción laboral de los mismos y la mejora en el rendimiento de los alumnos (OECD, 2009, 2014, 2019).

A diferencia de la autoeficacia, las creencias de eficacia colectiva han sido menos estudiadas debido a la complejidad del constructo y a la falta de medidas sólidas disponibles (Bandura, 1997). Sin embargo, distintos estudios afirman que a mayor nivel de eficacia colectiva docente, mayor es el rendimiento alcanzado por los alumnos (Bandura, 1993; Goddard, Skrla, \& Salloum, 2017; McCoach \& Colbert, 2010; Moolenaar, Sleegers, \& Daly, 2012). En esta linea, Goddard et al. (2000) afirman que las creencias que comparten los profesores dan forma a sus actuaciones y a la cultura del centro. Por eso en una escuela en la que los profesores comparten niveles altos de eficacia colectiva, se promueven expectativas mayores y un esfuerzo constante para seguir alcanzando las metas comunes (Goddard et al., 2017).

De ahí la importancia de conocer y fomentar el desarrollo de las creencias de eficacia colectiva de los profesores como medio para alcanzar el éxito educativo. Así pues, el presente estudio busca conocer las tendencias de las investigaciones realizadas con respecto a dichas creencias. Para esto se ha elegido realizar un mapeo sistemático con el fin 
de obtener una visión amplia y global del desarrollo y estudio de la eficacia colectiva docente en el ámbito académico.

\section{Método}

El mapeo sistemático es un método utilizado para conocer y contextualizar un determinado tema. Dicho método es considerado como un estudio secundario al tratarse de una revisión bibliográfica cuyo fin es identificar, evaluar y sintetizar información de diversas investigaciones con respecto a una temática y a unas preguntas previamente establecidas (Navarro Corona \& Ramírez Montoya, 2018). En algunas ocasiones el mapeo sistemático es utilizado como el primer paso para la realización de una revisión sistemática, ya que ofrece un resumen visual y global de la manera en que un tema ha sido abordado y estudiado (Petersen, Feldt, Mujtaba, \& Mattsson, 2008). De ahí que, a diferencia de las revisiones sistemáticas, el mapeo sistemático busque responder a preguntas más generales que permitan estructurar un área de investigación (Petersen, Vakkalanka, \& Kuzniarz, 2015).

Los mapeos sistemáticos se caracterizan por seguir una serie de pasos que guían su proceso de realización. Así pues, en la elaboración del presente mapeo se siguieron los pasos expuestos en la Figura 1.

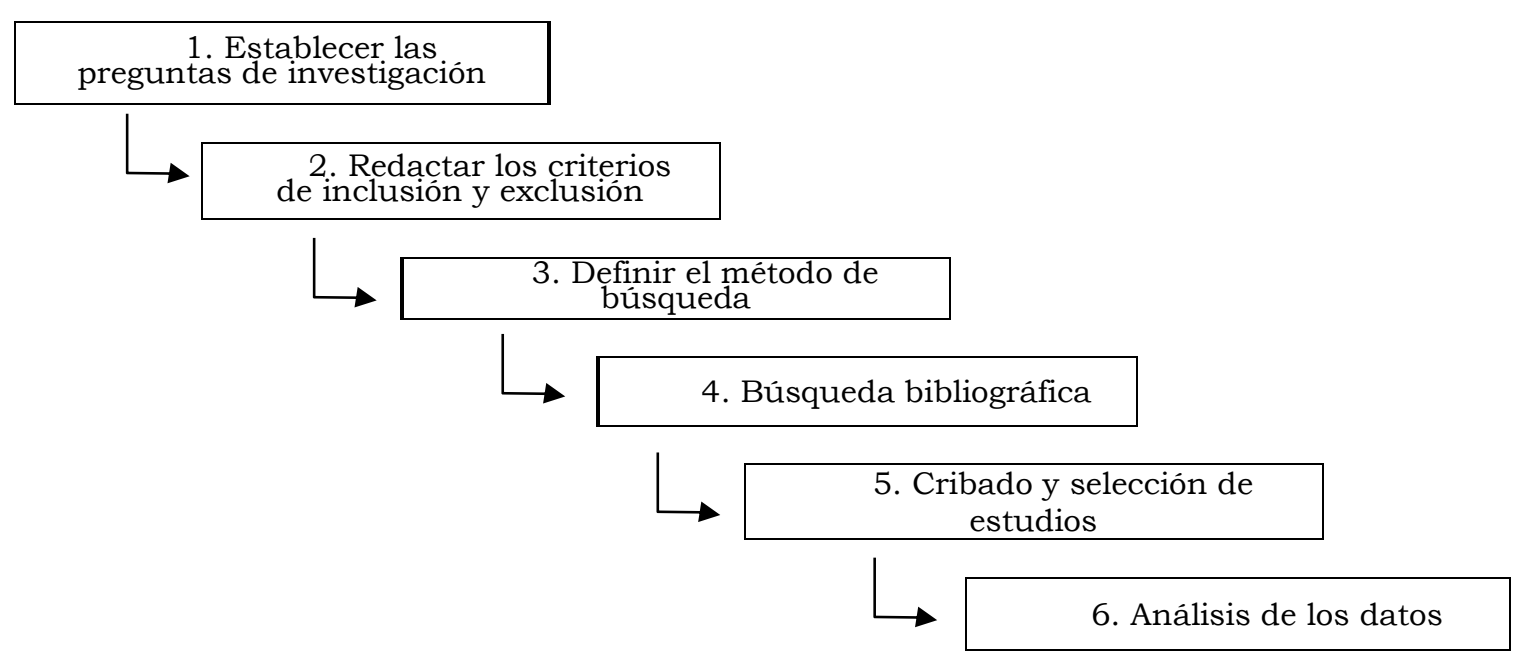

Figura 1. Pasos para la realización del mapeo sistemático

\section{Paso 1: Establecer las preguntas de investigación}


En primer lugar, se establecieron las preguntas de investigación que guiarán el presente estudio. Las preguntas son de carácter general debido a que el objetivo del mapeo sistemático es conocer las tendencias de investigación de los estudios publicados con respecto a la eficacia colectiva docente. La Tabla 1 muestra las seis preguntas de investigación.

Tabla 1

Preguntas de investigación

\begin{tabular}{cc}
\hline$\#$ & Pregunta \\
\hline 1 & ¿En qué años las bases de datos de Scopus y WoS recogen artículos de \\
2 & investigación sobre "collective teacher efficacy"? \\
3 & ¿Cuál es la distribución geográfica de los estudios? \\
4 & ¿Con qué variables se relaciona la eficacia colectiva docente? \\
5 & ¿Qué instrumentos se utilizan para evaluar la eficacia colectiva de los \\
6 & profesores?
\end{tabular}

\section{Paso 2: Redactar los criterios de inclusión y exclusión}

Una vez redactadas las preguntas de investigación se establecieron los criterios a tener en cuenta para la inclusión de estudios en el mapeo sistemático; así como también los criterios de exclusión que ayudarán a delimitar los resultados obtenidos de la búsqueda. En la Tabla 2 se presentan ambos tipos de criterios.

Tabla 2

Criterios de inclusión y exclusión

Criterios de inclusión Criterios de exclusión

Trabajos con "collective teacher efficacy" en el título, resumen o palabras clave

Trabajos duplicados en WoS

Trabajos publicados en un idioma distinto al español o al inglés

Trabajos de revisión bibliográfica y/o meta-análisis Trabajos no publicados como artículos de revista

Trabajos indexados en Scopus o WoS Trabajos en los que la eficacia colectiva docente no sea el objeto principal de estudio

\section{Paso 3: Definir el método de búsqueda}

Los estudios incluidos en la presente investigación fueron seleccionados durante el mes de septiembre de 2019. Las bases de datos consultadas fueron las siguientes:

- Scopus: es una de las mayores bases de datos de literatura revisada por pares. 
- Web of Science (WoS): ofrece una amplia variedad de documentos pertenecientes a diversas revistas de prestigio en el mundo de la investigación en Ciencias Sociales.

Por su parte, los términos utilizados para realizar las búsquedas se redujeron al constructo de interés, es decir, al de eficacia colectiva docente. El término fue utilizado en inglés, collective teacher efficacy, debido a que se realizó una búsqueda previa con el constructo en español y no se encontraron resultados. Así pues, con el fin de ampliar horizontes y encontrar un mayor número de estudios, se utilizaron las cadenas de búsqueda booleanas expuestas en la Tabla 3. En cuanto al periodo de tiempo comprendido en la búsqueda, no se estableció un límite en el rango de años a incluir, sino que se dejó abierto para responder mejor a la primera pregunta de investigación planteada (ver Tabla 1).

Tabla 3

Cadenas de búsqueda booleanas

\begin{tabular}{cc}
\hline Base de datos & Cadenas de búsqueda booleanas \\
\hline Scopus & TITLE-ABS-KEY("collective teacher efficacy") \\
WoS & TS=("collective teacher efficacy") \\
\hline
\end{tabular}

\section{Paso 4: Búsqueda bibliográfica}

Como resultado de las búsquedas bibliográficas, se obtuvieron un total de 90 estudios cuyos datos e información fueron descargados e introducidos en un documento Excel. La Tabla 4 especifica qué información fue recogida para cada uno de los estudios encontrados.

Tabla 4

Información extraída para cada uno de los estudios

\begin{tabular}{c}
\hline Información extraída \\
Autor(es) \\
Titulo \\
Referencia formato APA \\
Año \\
Base de datos \\
Revista \\
Número de citas \\
Abstract \\
Palabras clave del autor(es) \\
Tipo de documento \\
Distribución geográfica \\
Variables estudiadas
\end{tabular}




\section{Nivel escolar}

Instrumento utilizado para evaluar la eficacia colectiva docente

\section{Paso 5: Cribado y selección de estudios}

Después de descargar y extraer la información de los 90 estudios, se procedió a la aplicación de los criterios de exclusión presentados anteriormente en la Tabla 2. Así pues, se eliminaron en primer lugar los trabajos duplicados en la base de datos de Web of Science, reduciendo el número de estudios a un total de 60. Después, se excluyeron también aquellos trabajos publicados en otro idioma distinto al español o al inglés; de carácter teórico al tratarse de una revisión; que no hayan sido publicados como artículos de revista; y cuyo objeto principal de estudio sea otro distinto al de la eficacia colectiva docente. Finalmente, los 35 estudios incluidos en el mapeo fueron numerados con el objetivo de facilitar su localización y favorecer el análisis de los mismos (ver Anexo 1). La Figura 2 presenta el diagrama de bloques que refleja el proceso de selección de los estudios; mientras que la Tabla 5 muestra el número de estudios descartados del mapeo sistemático según los criterios de exclusión.

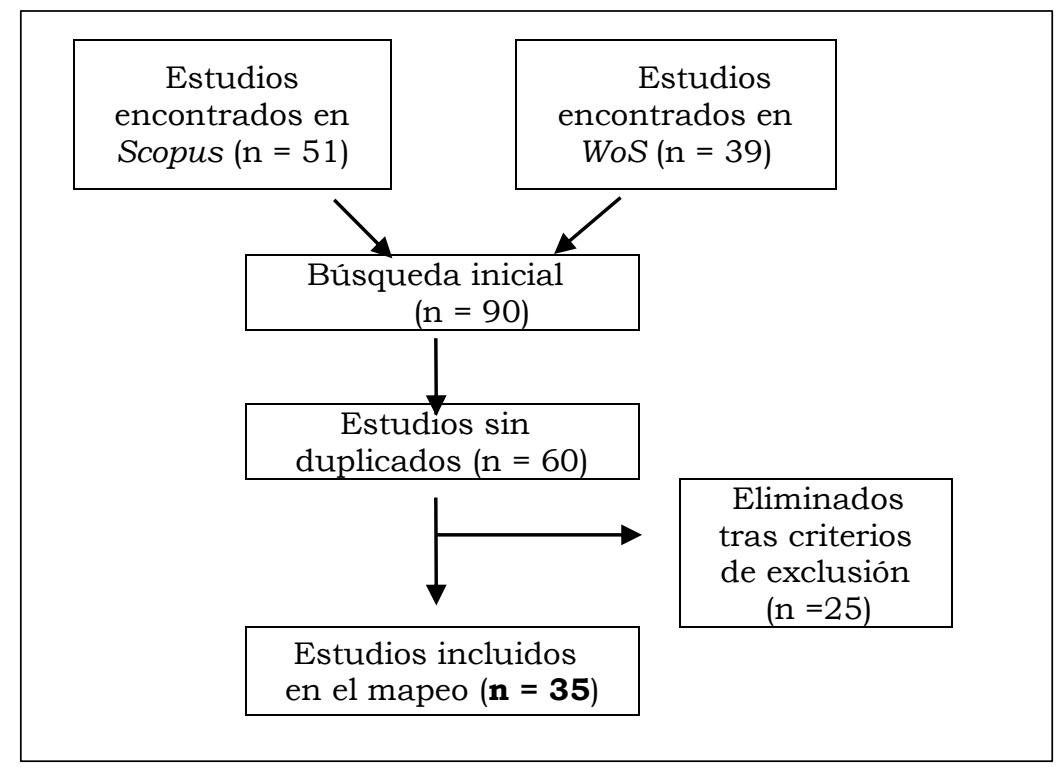

Figura 2. Diagrama de bloques sobre la selección de estudios incluidos en el mapeo sistemático 
Tabla 5

Número de estudios excluidos según los criterios establecidos

\begin{tabular}{cc}
\hline Criterios de exclusión & $\begin{array}{c}\text { Número de } \\
\text { estudios excluidos }\end{array}$ \\
\hline $\begin{array}{c}\text { Trabajos publicados en un idioma distinto al } \\
\text { español o al inglés }\end{array}$ & 2 \\
Trabajos de revisión bibliográfica y/o meta- \\
$\begin{array}{c}\text { análisis } \\
\text { Trabajos no publicados como artículos de } \\
\text { revista }\end{array}$ & 5 \\
Trabajos en los que la eficacia colectiva \\
docente no sea el objeto principal de estudio
\end{tabular}

\section{Paso 6: Análisis de los datos}

Una vez aplicados los criterios de inclusión y exclusión se procedió a analizar los datos obtenidos para cada uno de los 35 estudios incluidos, con el objetivo de responder a las preguntas de investigación previamente planteadas.

\section{Resultados}

P1. ¿En qué años las bases de datos de Scopus y WoS recogen artículos de investigación sobre "collective teacher efficacy"?

Los resultados obtenidos en las búsquedas fueron muy interesantes debido a que muestran claramente el momento en que el concepto de eficacia colectiva docente surgió como tal en las investigaciones. En este sentido, se puede decir que se trata de un constructo bastante reciente cuya aparición se encuentra en el año 2000 y cuyo momento de mayor auge parece ser el actual. En la Figura 3 se observa el número de publicaciones por año, ilustrando así el interés y producción de los estudios sobre la eficacia colectiva de los profesores en revistas indexadas en las bases de datos de Scopus y WoS. 


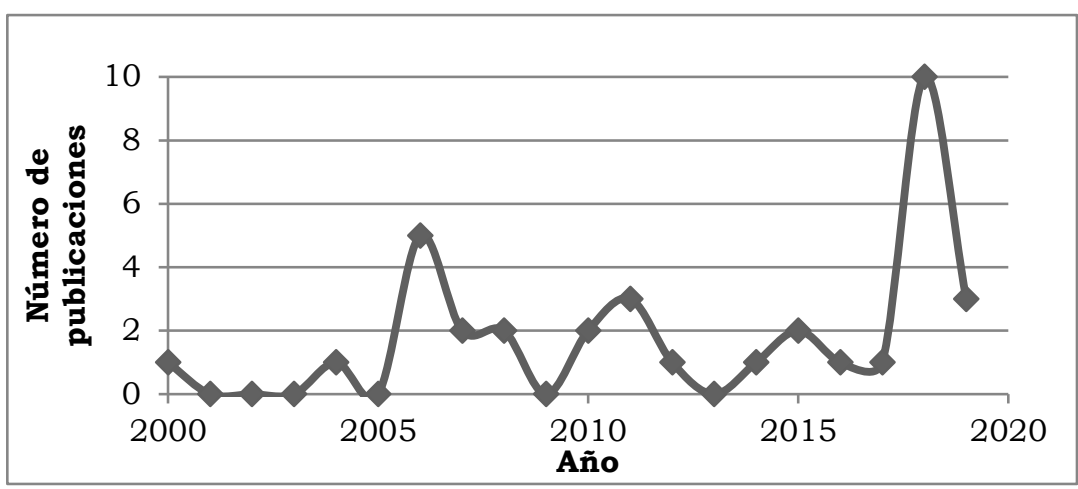

Figura 3. Producción de estudios sobre la eficacia colectiva docente

P2. ¿Cuál es la distribución geográfica de los estudios?

La producción científica sobre la eficacia colectiva proviene de catorce países distintos, tal como se ilustra en la Figura 4. En la Tabla 6 se muestra el número de estudios encontrados por país. Los países que más han realizado y publicado estudios al respecto son Estados Unidos, Irán y Turquía; con ocho, seis y cuatro artículos respectivamente. Resulta llamativo el hecho de que no aparezca ningún estudio realizado en países de habla hispana y que todos los estudios incluidos en el mapeo sistemático hayan sido publicados en inglés. Lo cual refleja un cierto dominio de los países anglosajones con respecto a la temática de interés, proporcionando el $34 \%$ de los estudios incluidos.

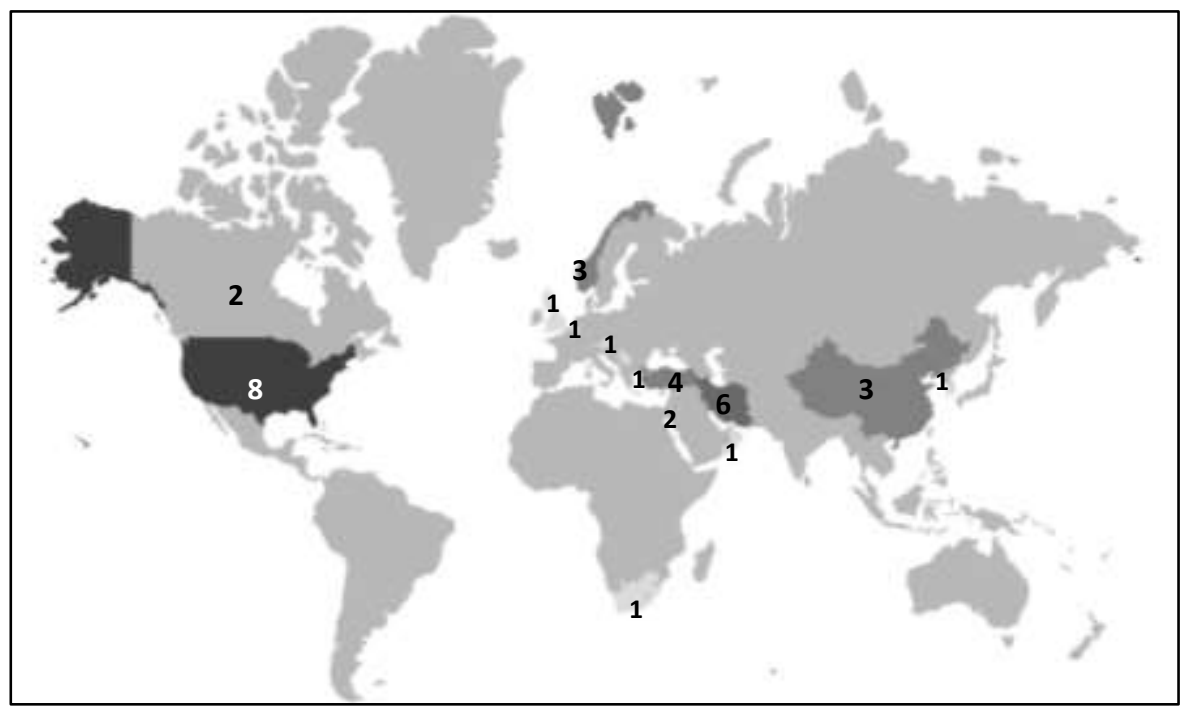

Figura 4. Distribución geográfica de los estudios 
Tabla 6

Estudios incluidos por pais

\begin{tabular}{|c|c|c|c|c|c|c|c|}
\hline País & $\begin{array}{c}\text { Número de } \\
\text { estudios }\end{array}$ & Año & Ref. art. & País & $\begin{array}{c}\text { Número de } \\
\text { estudios }\end{array}$ & Año & Ref. art. \\
\hline \multirow{8}{*}{ EE.UU.* } & \multirow[t]{8}{*}{ 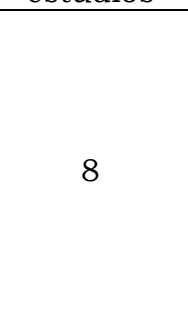 } & 2000 & 14 & \multirow{2}{*}{ China } & \multirow{2}{*}{3} & 2011 & 20 \\
\hline & & 2004 & 19 & & & 2019 & 7,22 \\
\hline & & 2006 & 3 & \multirow{3}{*}{ Noruega } & \multirow{3}{*}{3} & 2007 & 31 \\
\hline & & 2007 & 35 & & & 2010 & 32 \\
\hline & & 2008 & 16 & & & 2015 & 33 \\
\hline & & 2010 & 23 & Canadá* & 2 & 2006 & 28,29 \\
\hline & & 2011 & 13 & \multirow{2}{*}{ Israel } & \multirow{2}{*}{2} & 2006 & 30 \\
\hline & & 2018 & 10 & & & 2019 & 27 \\
\hline \multirow{3}{*}{ Irán } & \multirow{3}{*}{6} & 2016 & 5 & Austria & 1 & 2018 & 17 \\
\hline & & 2017 & 34 & Bélgica & 1 & 2015 & 6 \\
\hline & & 2018 & $\begin{array}{c}1,2,12 \\
15\end{array}$ & Corea & 1 & 2014 & 21 \\
\hline \multirow{4}{*}{ Turquía } & \multirow{4}{*}{4} & 2008 & 11 & Omán & 1 & 2018 & 4 \\
\hline & & 2011 & 18 & Reino Unido* & 1 & 2006 & 26 \\
\hline & & 2012 & 8 & Serbia & 1 & 2018 & 25 \\
\hline & & 2018 & 9 & Sudáfrica* & 1 & 2018 & 24 \\
\hline
\end{tabular}

Nota. Los países anglosajones han sido marcados con un asterisco $\left(^{*}\right)$.

P3. ¿Cuáles son los artículos más citados?

Según las bases de datos consultadas, Scopus y WoS, el 17\% de los artículos no ha sido citado en otros trabajos de investigación, el 14\% ha sido citado una vez y el $69 \%$ restante ha sido citado al menos dos veces. En la Tabla 7 se muestra el número de veces que cada artículo ha sido citado y en la Tabla 8 se presenta información más específica de los tres artículos más citados. Sobresale el hecho de que dos de ellos sean estudios realizados en Noruega por los autores Skaalvik y Skaalvik.

Tabla 7

Número de citas por estudio

\begin{tabular}{cc|cc}
\hline Número de citas & Ref. art. & Número de citas & Ref. art. \\
\hline 0 & $1,2,10,12,22,34$ & 21 & 19 \\
\hline 1 & $7,9,17,24,27$ & 22 & 3 \\
\hline 2 & 25 & 30 & 11 \\
\hline 3 & 5 & 67 & 20 \\
\hline 7 & 4 & 76 & 28 \\
\hline 8 & 15 & 92 & 35 \\
\hline 9 & 6,23 & 116 & 29 \\
\hline 14 & 21 & 144 & 16 \\
\hline 16 & 8,30 & 327 & 32 \\
\hline 17 & 18,26 & 408 & 14 \\
\hline 18 & 13 & 477 &
\end{tabular}


Tabla 8

Información de los artículos con mayor número de citas

\begin{tabular}{|c|c|c|c|c|c|c|}
\hline Ref. art. & Autores & Año & País & Revista & $\begin{array}{c}\text { Cuartil } \\
\text { (SJR) }\end{array}$ & $\begin{array}{l}\text { Número } \\
\text { de citas }\end{array}$ \\
\hline 14 & $\begin{array}{l}\text { Goddard, Hoy y } \\
\text { Woolfolk Hoy }\end{array}$ & 2000 & EE.UU. & $\begin{array}{c}\text { American } \\
\text { Educational } \\
\text { Research } \\
\text { Journal }\end{array}$ & Q1 & 477 \\
\hline 31 & $\begin{array}{l}\text { Skaalvik y } \\
\text { Skaalvik }\end{array}$ & 2007 & Noruega & $\begin{array}{l}\text { Journal of } \\
\text { Educational } \\
\text { Psychology }\end{array}$ & Q1 & 408 \\
\hline 32 & $\begin{array}{l}\text { Skaalvik y } \\
\text { Skaalvik }\end{array}$ & 2010 & Noruega & $\begin{array}{c}\text { Teaching and } \\
\text { Teacher } \\
\text { Education }\end{array}$ & Q1 & 327 \\
\hline
\end{tabular}

P4. ¿Con qué variables se relaciona la eficacia colectiva docente?

Debido a las escasas investigaciones sobre la eficacia colectiva docente, uno de los objetivos del mapeo sistemático era conocer las variables con las que se le ha relacionado en otros estudios. De ahí que la Tabla 9 presenta las distintas variables familiarizadas con las creencias de eficacia colectiva docente y organizadas según el nivel al que corresponden. Como era de esperar, aparecen variables como el índice socioeconómico, el rendimiento del alumno y las creencias de autoeficacia docente. Sin embargo, variables como el liderazgo del director, tanto instruccional como transformacional, presentan una alta aparición en estudios sobre la eficacia colectiva. Asimismo, variables del profesorado como el burnout y el compromiso o la implicación docente sobresalen en diversas investigaciones.

Tabla 9

Variables relacionadas con la eficacia colectiva docente

\begin{tabular}{cccc}
\hline \multirow{2}{*}{ Nivel } & Variable & \multicolumn{1}{c}{$\begin{array}{c}\text { Número } \\
\text { de } \\
\text { estudios }\end{array}$} & Ref. artículo \\
\hline \multirow{2}{*}{ Contextual } & Nivel socioeconómico & 6 & $3,6,13,14,23,26$ \\
\cline { 2 - 4 } & Escuelas urbanas y suburbanas & 2 & 16,30 \\
\cline { 2 - 4 } & Clima organizacional & 2 & 21,34 \\
\cline { 2 - 4 } & Visión compartida & 2 & 19,27 \\
\cline { 2 - 4 } Escolar & Comunidad escolar & 1 & 28 \\
\cline { 2 - 4 } & Clima de colaboración & 1 & 3 \\
\cline { 2 - 4 } & Nivel escolar & 1 & 6 \\
\cline { 2 - 4 } & Capital social & 1 & 24 \\
\cline { 2 - 4 } & Escuelas de bajo rendimiento & 1 & 3 \\
\cline { 2 - 4 } & Estructura escolar habilitadora & 1 & 3 \\
\cline { 2 - 4 } & Escuela internacional & 1 & 3 \\
\hline
\end{tabular}


Mapeo sistemático de la literatura sobre la eficacia colectiva docente

\begin{tabular}{|c|c|c|c|}
\hline \multirow{5}{*}{ Directivo } & Liderazgo transformacional & 6 & $11,18,22,25,28,29$ \\
\hline & Liderazgo instruccional & 6 & $4,8,9,13,15,27$ \\
\hline & Liderazgo del director & 1 & 35 \\
\hline & Confianza director-profesor & 1 & 33 \\
\hline & Autoeficacia del director & 1 & 15 \\
\hline \multirow{17}{*}{ Profesorado } & Autoeficacia docente & 14 & $\begin{array}{c}8,9,10,11,13,16 \\
18,19,24,25,31,32, \\
34,35\end{array}$ \\
\hline & $\begin{array}{l}\text { Compromiso/implicación del } \\
\text { profesor }\end{array}$ & 6 & $4,15,20,29,33,35$ \\
\hline & Enseñanza del inglés & 3 & $1,2,12$ \\
\hline & Burnout del profesorado & 3 & $21,31,32$ \\
\hline & $\begin{array}{l}\text { Compromiso con la misión } \\
\text { escolar }\end{array}$ & 2 & 15,28 \\
\hline & Control externo & 2 & 31,32 \\
\hline & $\begin{array}{l}\text { Enseñanza culturalmente } \\
\text { receptiva }\end{array}$ & 2 & 10,12 \\
\hline & Expectativas de resultados & 1 & 10 \\
\hline & Satisfacción laboral & 1 & 32 \\
\hline & $\begin{array}{l}\text { Comunidades profesionales de } \\
\text { aprendizaje }\end{array}$ & 1 & 20 \\
\hline & Confianza del profesorado & 1 & 20 \\
\hline & Profesores nóveles & 1 & 33 \\
\hline & Abandono de la profesión docente & 1 & 27 \\
\hline & Empoderamiento del profesor & 1 & 5 \\
\hline & Co-enseñanza & 1 & 17 \\
\hline & Profesorado en prácticas & 1 & 16 \\
\hline & Años de experiencia laboral & 1 & 12 \\
\hline \multirow[t]{2}{*}{ Alumnado } & Rendimiento académico & 7 & $\begin{array}{c}6,13,14,23,24,26 \\
28\end{array}$ \\
\hline & Implicación del alumno & 1 & 34 \\
\hline
\end{tabular}

P5. ¿Qué instrumentos se utilizan para evaluar la eficacia colectiva de los profesores?

La sexta pregunta de investigación busca conocer qué instrumentos han sido los más utilizados para evaluar y medir las creencias de eficacia colectiva docente. El interés por esta cuestión se debe a que cada instrumento se fundamenta en una manera determinada de concebir dicho constructo. La Tabla 10 muestra cuáles fueron los instrumentos utilizados para cada uno de los artículos incluidos en el presente mapeo. 
Tabla 10

Instrumentos utilizados para evaluar las creencias de eficacia

\begin{tabular}{|c|c|c|c|c|}
\hline Instrumento & $\begin{array}{l}\text { Número de } \\
\text { estudios }\end{array}$ & Porcentaje & País & Ref. artículo \\
\hline \multirow{5}{*}{$\begin{array}{l}\text { Collective Teacher Efficacy Scale } \\
\text { (CTE Scale; Goddard et al., 2000) }\end{array}$} & \multirow{5}{*}{12} & \multirow{5}{*}{$34 \%$} & $\begin{array}{l}\text { EE.UU. } \\
\text { Turquia }\end{array}$ & $\begin{array}{l}3,14,19,23 \\
8,9,18\end{array}$ \\
\hline & & & Canadá & 28,29 \\
\hline & & & Corea & 21 \\
\hline & & & Irán & 12 \\
\hline & & & Omán & 4 \\
\hline \multirow{5}{*}{$\begin{array}{c}\text { Collective Efficacy Scale } \\
\text { (CE-Scale; Goddard, 2002) }\end{array}$} & \multirow{5}{*}{7} & \multirow{5}{*}{$20 \%$} & EE.UU. & 13,16 \\
\hline & & & China & 7,22 \\
\hline & & & Bélgica & 6 \\
\hline & & & Irán & 5 \\
\hline & & & Sudáfrica & 24 \\
\hline \multirow{4}{*}{$\begin{array}{l}\text { Collective Teacher Belief Scale } \\
\text { (CTBS; Tschannen-Moran \& Barr, } \\
\text { 2004) }\end{array}$} & \multirow{4}{*}{6} & \multirow{4}{*}{$17 \%$} & Irán & 15,34 \\
\hline & & & Israel & 27,30 \\
\hline & & & China & 20 \\
\hline & & & Reino Unido & 26 \\
\hline \multirow{2}{*}{$\begin{array}{c}\text { Perceived Collective Teacher } \\
\text { Efficacy Scale (Skaalvik \& } \\
\text { Skaalvik, 2007) }\end{array}$} & \multirow[b]{2}{*}{4} & \multirow[b]{2}{*}{$11,5 \%$} & Noruega & $31,32,33$ \\
\hline & & & Serbia & 25 \\
\hline \multirow{3}{*}{ Desarrollo del propio instrumento } & \multirow{3}{*}{4} & \multirow{3}{*}{$11,5 \%$} & EE.UU. & 10,35 \\
\hline & & & Irán & 1 \\
\hline & & & Turquía & 11 \\
\hline $\begin{array}{c}\text { Collective teacher self-efficacy } \\
\text { expectations (Schwarzer \& } \\
\text { Jerusalem, 2002) }\end{array}$ & 1 & $3 \%$ & Austria & 17 \\
\hline Entrevista & 1 & $3 \%$ & Irán & 2 \\
\hline
\end{tabular}

P6. ¿En qué niveles educativos se desarrollan los estudios?

Otra consideración a tener en cuenta en el estudio de la eficacia colectiva docente, es el nivel educativo sobre el que se realiza la investigación. La Tabla 11 especifica los niveles escolares en los que los estudios fueron efectuados. Tal y como ahí se observa, sorprende que el $40 \%$ de los estudios incluidos en el mapeo sistemático fueron realizados con profesores de primaria.

Tabla 11

Clasificación de artículos según el nivel escolar

\begin{tabular}{cccc}
\hline Nivel escolar & $\begin{array}{c}\text { Número de } \\
\text { estudios }\end{array}$ & Porcentaje & Ref. artículo \\
\hline \multirow{2}{*}{ Educación Primaria } & 14 & $40 \%$ & $\begin{array}{c}4,6,8,11,14,15,18, \\
22,26,27,28,29,30, \\
34\end{array}$ \\
\hline
\end{tabular}


Mapeo sistemático de la literatura sobre la eficacia colectiva docente

\begin{tabular}{|c|c|c|c|c|}
\hline & ducación Secundaria & 3 & $8 \%$ & $17,21,25$ \\
\hline & Bachillerato & 2 & $6 \%$ & 13,19 \\
\hline & rimaria + Secundaria & 4 & $12 \%$ & $20,31,32,33$ \\
\hline & $\begin{array}{l}\text { imaria + Secundaria }+ \\
\text { Bachillerato } \\
\end{array}$ & 3 & $8 \%$ & $3,7,9$ \\
\hline & No especifica & 4 & $12 \%$ & $5,23,24,35$ \\
\hline \multirow{3}{*}{ Otros } & Educación especial & 1 & $3 \%$ & 10 \\
\hline & Instituto de idiomas & 3 & $8 \%$ & $1,2,12$ \\
\hline & $\begin{array}{c}\text { Profesorado en } \\
\text { prácticas }\end{array}$ & 1 & $3 \%$ & 16 \\
\hline
\end{tabular}

\section{Discusión}

La búsqueda de la mejora educativa es un tema constante en el ámbito de la educación. Las creencias de eficacia colectiva docente se presentan como una variable a nivel grupal que forma parte de la cultura operativa del centro y cuyas repercusiones se observan en distintos niveles. Por su parte, el mapeo sistemático es una excelente herramienta para establecer un primer contacto con un determinado tema. En este caso, su uso nos ha permitido tomar una fotografia actual de la manera en que las creencias de eficacia colectiva docente han sido abordadas y estudiadas. En concreto, las seis preguntas de investigación nos han permitido ahondar en distintos aspectos, arrojándonos luz acerca de lo ya investigado y de lo que queda por investigar.

Tal y como se dijo en un principio, el objetivo de este pequeño estudio era conocer las tendencias de las investigaciones realizadas con respecto a la eficacia colectiva docente. De ahí que las búsquedas fueron efectuadas de la manera más general posible, sin incluir un límite en el periodo de tiempo a estudiar. Sin embargo, algunas limitaciones deben ser reconocidas. En primer lugar, las búsquedas fueron únicamente realizadas en dos bases de datos, Scopus y WoS, por lo que el número de estudios encontrados no fue muy extenso. En segundo lugar, solamente se utilizó el término de "collective teacher efficacy", dejando fuera a todos aquellos estudios que no incluyeran de manera exacta dicho constructo en su título, resumen o palabras clave.

No obstante, esta última limitación nos permitió corroborar dónde y cuándo surgieron las investigaciones con dicho término para estudiar las creencias de capacidad de los profesores a nivel de centro. En este sentido, la Tabla 6 nos permite realizar un recorrido geográfico y temporal del estudio de la eficacia colectiva docente. El primer estudio encontrado [14] aparece en el año 2000 y se corresponde con el desarrollo y validación de una de las escalas más utilizadas actualmente para evaluar la eficacia colectiva: la Collective Teacher Efficacy Scale de Goddard, Hoy y Woolfolk 
Hoy. Asimismo, se observa claramente que el interés por esta temática surge en los países de habla inglesa, presentando diversos estudios entre los años 2000 y 2008 [3, 14, 16, 19, 26, 28, 29, 35]. No obstante, en el 2018 el interés por la eficacia colectiva docente trasciende a los distintos continentes y presenta un gran incremento en la producción científica. De hecho, el 37\% de los estudios incluidos en este mapeo sistemático han sido realizados en 2018 y 2019. Este aumento en el interés probablemente se deba a los estudios realizados por John Hattie (2016), quien afirma que la eficacia colectiva docente presenta un tamaño del efecto de 1.57 sobre el rendimiento de los alumnos.

Estados Unidos se muestra como el país con mayor número de contribuciones académicas. De igual manera, países como Irán $[1,2,5,12$, 15, 34], Turquía [8, 9, 11, 18] y Noruega [31, 32, 33] muestran un interés sólido y constante en el estudio de las creencias de eficacia del profesorado. En concreto, los investigadores noruegos Skaalvik y Skaalvik se presentan como los autores con mayor impacto en la comunidad científica por el alto número de citas en sus estudios [31,32]. No obstante, el artículo más citado es el de los autores Goddard et al. (2000) [14], debido probablemente a la fama y uso del instrumento que ahí se presenta.

En cuanto a las variables relacionadas con la eficacia colectiva docente, se observa una clara relación entre dicho constructo y las creencias de autoeficacia de los profesores. En concreto, el $40 \%$ de los artículos incluidos estudia las creencias de eficacia a nivel individual y grupal [8, 9, $10,11,13,16,18,19,24,25,31,32,34,35]$. Otra variable que sobresale por su estrecha relación es la del liderazgo del director, ya sea de tipo transformacional $[11,18,22,25,28,29]$ o instruccional $[4,8,9,13,15$, 27]. Varios de estos estudios proponen la eficacia colectiva docente como variable mediadora entre el liderazgo del director y el rendimiento del alumno [13, 18, 28]; argumentando que el director impacta al alumno en la medida en que contribuye al desarrollo de las creencias de eficacia colectiva en sus profesores.

Por su parte, las variables a nivel del profesorado resultan mucho más numerosas, abarcando factores relacionados con el bienestar socioemocional de los profesores -satisfacción laboral y burnout-, como con el compromiso con la profesión docente, con la misión escolar y con el alumnado. Así pues, los estudios muestran que a mayor nivel de eficacia colectiva mayores son sus niveles de implicación [4, 15, 20, 29, 33, 35], de satisfacción laboral [32], y de autoeficacia docente [8, 9, 10, 11, 13, 16, 18, 19, 24, 25, 31, 32, 34, 35]; siendo menor el indice de burnout en el profesorado [36, 50, 51]. Asimismo, tal como Hattie (2016) defiende, la eficacia colectiva docente repercute de manera clara en el rendimiento académico [6, 13, 14, 23, 24, 26, 28], llegando incluso a superar el impacto del nivel socioeconómico. En palabras de Donohoo (2017) "la eficacia colectiva docente, como una influencia en el rendimiento del alumno, es 
una contribución que proviene de la escuela, y no de los hogares ni de los alumnos" (p. 5 [traducción propia]). De ahí la importancia de su estudio y promoción.

Finalmente, en cuanto a aspectos más prácticos, la eficacia colectiva docente ha sido evaluada principalmente con los instrumentos desarrollados por Goddard (2002) y sus colaboradores (Goddard et al., 2000). De hecho el 54\% de los estudios incluidos en este mapeo (ver Tabla 10) han utilizado la CTE Scale o su versión corregida, la CE-Scale. Por su parte, el $17 \%$ de los estudios han utilizado la escala desarrollada por Tschannen-Moran y Barr (2004), la Collective Teacher Belief Scale, la cual surgió con el objetivo de dar mayor solidez a la medición de las creencias de eficacia colectiva de los profesores. Por último la escala de Skaalvik y Skaalvik (2007), la Perceived Collective Teacher Efficacy Scale, representa el $11,5 \%$ de los estudios, siendo utilizada en aquellas investigaciones correspondientes a su país de origen, Noruega.

\section{Conclusiones}

Tras la realización de este mapeo sistemático, se espera haber conseguido una visión histórica y actual del estudio de las creencias de eficacia colectiva docente. El interés por esta temática se debe tanto al fuerte impacto que tienen estas creencias sobre el aprendizaje de los alumnos, como a la necesidad de indagar más acerca de variables relacionadas con la mejora educativa.

En primer lugar, se observa un crecimiento en el interés por la eficacia colectiva docente después de los estudios realizados por Hattie (2016). En concreto, se observa que el interés aumenta y se expande por países de todo el mundo y no solamente en el contexto anglosajón. Sin embargo, sorprende que dicho crecimiento no se vea reflejado en países iberoamericanos. Por lo que sería interesante investigar si los países hispanohablantes cuentan con herramientas válidas y fiables para evaluar la eficacia colectiva de los profesores. Ya que, como se mencionó al principio, la medición de esta variable es compleja y no siempre se cuenta con medidas sólidas para estudiarla (Bandura, 1997).

En segundo lugar, se observan los avances y las líneas en proceso de crecimiento, tales como el papel del liderazgo educativo en la potenciación de las creencias de eficacia de los profesores; o el desarrollo de dichas creencias en ámbitos educativos concretos -educación especial y enseñanza del inglés- o con futuros docentes, lo que en inglés se conoce como pre-service teachers.

A pesar de que el número de estudios encontrados fue limitado, se observa un claro impacto de las creencias de eficacia colectiva docente sobre el aprendizaje y rendimiento de los alumnos. Así como también, una 
relación positiva con variables como la satisfacción laboral, la autoeficacia docente y el aumento en el compromiso e implicación de los profesores con respecto a su labor profesional. Sin embargo, se echa en falta investigaciones centradas en conocer cómo se pueden fomentar y desarrollar las creencias de eficacia docente, tanto a nivel individual como a nivel grupal. En este sentido, futuras investigaciones podrian ahondar en esta temática, con el objetivo de ofrecer herramientas concretas para la formación docente y la práctica educativa.

Asimismo, si se toma en cuenta la estrecha relación encontrada entre la eficacia colectiva de los profesores y el liderazgo educativo, habría que plantearse una posible formación de directivos que promueva aspectos como la colaboración docente, la confianza, el empoderamiento de los profesores, el desarrollo de una visión compartida, entre otros. El director debe tomar un rol activo en la potenciación de estas creencias, ya que los profesores de todos los centros comparten juicios acerca de su capacidad o no para hacer una mejora en el alumno. Lo importante aquí es que esas creencias sean una herramienta que impulse la mejora de los docentes y, consecuentemente, la mejora de los discentes.

En definitiva, se percibe un redescubrimiento y un interés creciente por el desarrollo y la promoción de la eficacia colectiva docente como un medio para alcanzar la eficacia escolar en todo sus niveles. El vacío en el ámbito hispano es patente, pero se cuenta con la ventaja de tener un trayecto recorrido en la esfera anglosajona e internacional. La realización del presente estudio intentó mostrar de manera más clara dicho recorrido con el fin de conocer qué es lo que se ha investigado y qué es lo que falta por investigar, para así centrar mejor los futuros estudios sobre la eficacia colectiva de los profesores.

\section{Agradecimientos}

Los autores agradecen los comentarios de los revisores, así como la financiación recibida por el Programa de Ayudas de la Asociación de Amigos de la Universidad de Navarra.

\section{Referencias}

Bandura, A. (1986). Social Foundations of Thought and Action: A Social Cognitive Theory. Englewood Cliffs, N.J.: Prentice-Hall.

Bandura, A. (1993). Perceived Self-Efficacy in Cognitive Development and Functioning. Educational Psychologist, 28(2), 117-148. https://doi.org/10.1207/s15326985ep2802_3 
Bandura, A. (1997). Self-efficacy: the exercise of control. New York: W. H. Freeman.

Barber, M., \& Mourshed, M. (2007). How the World's Best-Performing School Systems Come Out On Top. McKinsey \& Company.

Darling-Hammond, L., Hyler, M. E., \& Gardner, M. (2017). Effective teacher professional development. Palo Alto, CA. Recuperado de https://learningpolicyinstitute.org/product/teacher-prof-dev

Donohoo, J. (2017). Collective efficacy: how educators' beliefs impact student learning. Thousand Oaks, CA: Corwin.

Eells, R. J. (2011). Meta-analysis of the relationship between collective teacher efficacy and student achievement. Loyola University.

Gibson, S., \& Dembo, M. H. (1984). Teacher efficacy: A construct validation. $\begin{array}{lll}\text { Journal of Educational Psychology, 76(4), 569-582. } & \text {. }\end{array}$ https://doi.org/10.1037/0022-0663.76.4.569

Goddard, R. D. (2002). A Theoretical and Empirical Analysis of the Measurement of Collective Efficacy: The Development of a Short Form. Educational and Psychological Measurement, 62(1), 97-110. https://doi.org/10.1177/0013164402062001007

Goddard, R. D., Hoy, W. K., \& Woolfolk Hoy, A. (2000). Collective Teacher Efficacy: Its Meaning, Measure, and Impact on Student Achievement. American Educational Research Journal, 37(2), 479-507. https://doi.org/10.3102/00028312037002479

Goddard, R. D., Skrla, L., \& Salloum, S. J. (2017). The Role of Collective Efficacy in Closing Student Achievement Gaps: A Mixed Methods Study of School Leadership for Excellence and Equity. Journal of Education for Students Placed at Risk, 22(4), 220-236. https://doi.org/ 10.1080/10824669.2017.1348900

Hargreaves, A., \& Fullan, M. (2014). Capital profesional. (C. Mimiaga, Ed.). Madrid: Ediciones Morata.

Hattie, J. (2016). Third Annual Visible Learning Conference (subtitled Mindframes and Maximizers). Washington, DC.

Martínez Orbegozo, E. F. (2019). El centro y las prácticas de colaboración docente como medios para la mejora educativa. Recuperado de 
http://documentos.fedea.net/pubs/eee/eee2019-26.pdf

McCoach, D. B., \& Colbert, R. D. (2010). Factors underlying the collective teacher efficacy scale and their mediating role in the effect of socioeconomic status on academic achievement at the school level. Measurement and Evaluation in Counseling and Development, 43(1), 3147. https://doi.org/10.1177/0748175610362368

Moolenaar, N. M., Sleegers, P. J. C., \& Daly, A. J. (2012). Teaming up: Linking collaboration networks, collective efficacy, and student achievement. Teaching and Teacher Education, 28, 251-262. https://doi.org/10.1016/j.tate.2011.10.001

Navarro Corona, C., \& Ramírez Montoya, M. S. (2018). Systematic mapping of literature on teacher evaluation (2013-2017). Educação e Pesquisa, 44, 1-22. https://doi.org/10.1590/S1678-4634201844185677

OECD. (2009). Creating Effective Teaching and Learning Environments: First Results from TALIS. https://doi.org/https://doi.org/10.1787/9789264068780-en

OECD. (2014). A Teachers' Guide to TALIS 2013: Teaching and Learning International Survey. TALIS, OECD Publishing. https://doi.org/10.1787/9789264216075-en

OECD. (2019). TALIS 2018 Results (Volume I): Teachers and School Leaders as Lifelong Learners. Paris. https://doi.org/https://doi.org/10.1787/1d0bc92a-en

Petersen, K., Feldt, R., Mujtaba, S., \& Mattsson, M. (2008). Systematic mapping studies in software engineering. 12th International Conference on Evaluation and Assessment in Software Engineering, EASE 2008, 110 .

Petersen, K., Vakkalanka, S., \& Kuzniarz, L. (2015). Guidelines for conducting systematic mapping studies in software engineering: An update. Information and Software Technology, 64, 1-18. https://doi.org/10.1016/j.infsof.2015.03.007

Schwarzer, R., \& Jerusalem, M. (2002). Das Konzept der Selbstwirksamkeit. In Selbstwirksamkeit Und Motivationsprozesse in Bildunginstitutionen. Zeitschrift für Pädagogik, 44, 54-82.

Skaalvik, E. M., \& Skaalvik, S. (2007). Dimensions of Teacher Self-Efficacy and Relations With Strain Factors, Perceived Collective Teacher Efficacy, 
and Teacher Burnout. Journal of Educational Psychology, 99(3), 611-625. https://doi.org/10.1037/0022-0663.99.3.611

Stewart, V. (2018). How teachers around the world learn. Educational Leadership, 28-35.

Tschannen-Moran, M., \& Barr, M. (2004). Fostering Student Learning: The Relationship of Collective Teacher Efficacy and Student Achievement. Leadership and Policy in Schools, 3(3), 189-209. https://doi.org/10.1080/15700760490503706 


\section{Anexo 1}

1. Abedini, F., Bagheri, M. S. \& Sadighi, F. (2018). Exploring Iranian collective teacher efficacy beliefs in different ELT settings through developing a context-specific English language teacher collective efficacy scale. Cogent Education. 5(1). https://doi.org/10.1080/2331186X.2018.1552340

2. Abedini, F., Bagheri, M. S., Sadighi, F., \& Yarmohammadi, L. (2018). The constituent elements of collective teacher efficacy beliefs and their contributing factors in different ELT educational contexts: A qualitative study. Cogent Social Sciences, 4(1). https: / /doi.org/ 10.1080/23311886.2018.1500768

3. Adams, C. M., \& Forsyth, P. B. (2006). Proximate sources of collective teacher efficacy. Journal of Educational Administration, 44(6), 625-642. https: / / doi.org/ 10.1108/09578230610704828

4. Al-Mahdy, Y. F. H., Emam, M. M., \& Hallinger, P. (2018). Assessing the contribution of principal instructional leadership and collective teacher efficacy to teacher commitment in Oman. Teaching and Teacher Education, 69, 191-201. https://doi.org/10.1016/j.tate.2017.10.007

5. Baleghizadeh S. \& Goldouz E. (2016). The relationship between Iranian EFL teachers' collective efficacy beliefs, teaching experience and perception of teacher empowerment. Cogent Education, 3, 115. http://dx.doi.org/10.1080/2331186X.2016.1223262

6. Belfi, B., Gielen, S., De Fraine, B., Verschueren, K., \& Meredith, C. (2015). School-based social capital: The missing link between schools' socioeconomic composition and collective teacher efficacy. Teaching and Teacher Education, 45 , 3344. http://dx.doi.org/10.1016/j.tate.2014.09.001

7. Blatti, T., Clinton, J., \& Graham, L. (2019). Exploring collective teacher efficacy in an international school in Shanghai. International Journal of Learning, Teaching and Educational Research, 18(6), 214-235. https://doi.org/10.26803/ijlter.18.6.13

8. Calik, T., Sezgin, F., Kavgaci, H., \& Kilinc, A.C. (2012). Examination of Relationships between Instructional Leadership of School Principals and Self-Efficacy of Teachers and Collective Teacher Efficacy. Educational Sciences: Theory \& Practice, 12(4), 2498-2504.

9. Cansoy, R. \& Parlar, H. (2018). Examining the relationship between school principals' instructional leadership behaviors, teacher selfefficacy, and collective teacher efficacy. International Journal of Educational Management. https://doi.org/10.1108/IJEM-04-20170089

10. Chu, S. Y., \& Garcia, S. B. (2018). Collective Teacher Efficacy and Culturally Responsive Teaching Efficacy of Inservice Special Education 
Teachers in the United States. Urban Education. https: / / doi.org/ 10.1177/0042085918770720

11. Demir, K. (2008). Transformational leadership and collective efficacy: The moderating roles of collaborative culture and teachers' self-efficacy. Egitim Arastirmalari - Eurasian Journal of Educational Research, 33, 93112.

12. Ebrahimimehr, A. A., Homayoun, M. R. \& Mansoorian, S. M. A. (2018). The relationship between collective teacher efficacy and their collective responsibility from a psychoanalytic health perspective. International Journal of Pharmaceutical Research.

13. Fancera, S. F. \& Bliss, J. R. (2011). Instructional Leadership Influence on Collective Teacher Efficacy to Improve School Achievement. Leadership and Policy in Schools, 10(3), 349-370. https: / / doi.org/ 10.1080/15700763.2011.585537

14. Goddard, R. D., Hoy, W. K., \& Woolfolk Hoy, A. (2000). Collective Teacher Efficacy: Its Meaning, Measure, and Impact on Student Achievement. American Educational Research Journal, 37(2), 479-507. https: / / doi.org/ 10.3102/00028312037002479

15. Hallinger, P., Hosseingholizadeh, R., Hashemi, N., \& Kouhsari, M. (2018). Do beliefs make a difference? Exploring how principal selfefficacy and instructional leadership impact teacher efficacy and commitment in Iran. Educational Management Administration \& Leadership, 46(5),

800-

819. https://doi.org/10.1177/1741143217700283

16. Knoblauch, D. \& Woolfolk Hoy, A. (2008). "Maybe I can teach those kids." The influence of contextual factors on student teachers' efficacy beliefs. Teaching and Teacher Education, 24(1), 166-179. https: / / doi.org/ 10.1016/j.tate.2007.05.005

17. Krammer, M., Gastager, A., Lisa, P., Gasteiger-Klicpera, B., \& Rossmann, P. (2018). Collective self-efficacy expectations in Co-teaching teams - what are the influencing factors?. Educational Studies, 44(1), 99-114. https://doi.org/10.1080/03055698.2017.1347489

18. Kurt, T., Duyar, I., \& Çalik, T. (2011). Are we legitimate yet?: A closer look at the casual relationship mechanisms among principal leadership, teacher self-efficacy and collective efficacy. Journal of Management Development, $31(1)$, 71-86. https: / / doi.org/ 10.1108/02621711211191014

19. Kurz, T. B., \& Knight, S. L. (2004). An exploration of the relationship among teacher efficacy, collective teacher efficacy, and goal consensus. Learning Environments Research, 7(2), 111-128. https: / / doi.org/ 10.1023/B:LERI.0000037198.37750.0e

20. Lee, J. C., Zhang, Z. \& Yin, H. (2011). A multilevel analysis of the impact of a professional learning community, faculty trust in colleagues 
and collective efficacy on teacher commitment to students. Teaching and Teacher Education, 820-830. https://doi.org/10.1016/j.tate.2011.01.006

21. Lim, S. \& Eo, S. (2014). The mediating roles of collective teacher efficacy in the relations of teachers' perceptions of school organizational climate to their burnout. Teaching and Teacher Education, 44, 138147. http://dx.doi.org/10.1016/j.tate.2014.08.007

22. Liu, P., Li, L., \& Wang, J. (2019) Understanding the relationship between transformational leadership and collective teacher efficacy in Chinese primary schools. International Journal of Leadership in Education. https://doi.org/10.1080/13603124.2019.1623922

23. McCoach, D. B., \& Colbert, R. D. (2010). Factors underlying the collective teacher efficacy scale and their mediating role in the effect of socioeconomic status on academic achievement at the school level. Measurement and Evaluation in Counseling and Development, 43(1), 3147. https://doi.org/10.1177/0748175610362368

24. Mosoge, M. J., Challens, B. H., \& Xaba, M. I. (2018). Perceived collective teacher efficacy in low performing schools. South African Journal of Education, 38(2). https://doi.org/10.15700/saje.v38n2a1153

25. Ninković, S. R., \& Knežević Florić, O. Č. (2018). Transformational school leadership and teacher self-efficacy as predictors of perceived collective teacher efficacy. Educational Management Administration \& Leadership, 46(1), 49-64. https://doi.org/10.1177/1741143216665842

26. Parker, K., Hannah, E., \& Topping, K. J. (2006). Collective teacher efficacy, pupil attainment and socio-economic status in primary school. Improving Schools, 9(2), 111-129. https: / / doi.org/10.1177/1365480206064965

27. Qadach, M., Schechter, C., \& Da'as, R. (2019). Instructional leadership and teacher's intent to leave: The mediating role of collective teacher efficacy and shared vision. Educational Management Administration

Leadership. https://doi.org/10.1177/1741143219836683

28. Ross, J. A. \& Gray, P. (2006). School leadership and student achievement: The mediating effects of teacher beliefs. Canadian Journal of Education, 29(3), 798-822. https://doi.org/10.2307/20054196

29. Ross, J. A. \& Gray, P. (2006). Transformational leadership and teacher commitment to organizational values: The mediating effects of collective teacher efficacy. School Effectiveness and School Improvement, 17(2), 179-199. https://doi.org/10.1080/09243450600565795

30. Schechter, C. \& Tschannen-Moran, M. (2006). Teachers' sense of collective efficacy: An international view. International Journal of Educational Management, 20(6), 480-489. https://doi.org/10.1108/09513540610683720 
31. Skaalvik E. M. \& Skaalvik S. (2007). Dimensions of Teacher SelfEfficacy and Relations With Strain Factors, Perceived Collective Teacher Efficacy, and Teacher Burnout. Journal of Educational Psychology, 99(3), 611-625. http://dx.doi.org/10.1037/0022-0663.99.3.611

32. Skaalvik, E. M. \& Skaalvik, S. (2010). Teacher self-efficacy and teacher burnout: A study of relations. Teaching and Teacher Education, 26(4), 1059-1069. https://doi.org/10.1016/j.tate.2009.11.001

33. Tiplic, D., Brandmo, C., \& Elstad, E. (2015). Antecedents of Norwegian beginning teachers' turnover intentions. Cambridge Journal of Education, 45(4), 451474. http://dx.doi.org/10.1080/0305764X.2014.987642

34. Veiskarami, H. A., Ghadampour, E., \& Mottaghinia, M. R. (2017). Interactions among School Climate, Collective Self-Efficacy, and Personal Self-Efficiency: Evidence from Education Institutions. International Journal of Economic Perspectives, 11(1), 481-488.

35. Ware H. \& Kitsantas A. (2007). Teacher and collective efficacy beliefs as predictors of professional commitment. Journal of Educational Research, 100(5), 303-310. https://doi.org/10.3200/JOER.100.5.303310 
